\title{
A Single Mutation Converts the Nucleotide Specificity of Phenol Sulfotransferase from PAP to $\mathrm{AMP}^{\dagger}$
}

\author{
Yu-Shan Hsiao and Yuh-Shyong Yang* \\ Department of Biological Science and Technology, College of Science, National Chiao Tung University, Hsinchu, Taiwan, ROC
}

Received May 12, 2002; Revised Manuscript Received September 1, 2002

\begin{abstract}
Sulfotransferases (STs) catalyze all the known biological sulfonations, in which a sulfuryl group from a common sulfonate donor such as 3'-phosphoadenosine 5'-phosphosulfate (PAPS) is transferred to a nucleophilic acceptor. In addition to PAPS, phenol sulfotransferase (PST), a member of the ST family, utilizes other nucleotides as substrates with much less catalytic efficiency [Lin, E. S., and Yang, Y. S. (2000) Biochem. Biophys. Res. Commun. 271, 818-822]. Six amino acid residues of PST have been chosen for mutagenesis studies on the basis of a model of PST and its sequence alignment with those of available cytosolic and membrane-anchored STs. Systematic analyses of the mutants reveal that Ser134 is important for the regulation of nucleotide specificity between $3^{\prime}$-phosphoadenosine $5^{\prime}$-phosphate (PAP) and adenosine $5^{\prime}$-monophosphate (AMP). Kinetic studies also indicate that Ser134 plays a key role in nucleotide binding $\left(K_{\mathrm{m}}\right)$ but not in catalysis $\left(k_{\mathrm{cat}}\right)$. Consequently, the catalytic efficiency $\left(k_{\mathrm{cat}} / K_{\mathrm{m}}\right)$ of PST can be altered by 5 orders of magnitude with a mutation of Ser134. Moreover, the change in nucleotide specificity from PAP to AMP can be achieved by mutation of Ser134 to any of the following residues: Glu, Gln, Arg, and His. Roles of Lys44, Arg126, and Arg253, which interact directly with the 5'- and 3 '-phosphate of PAP, were also investigated by mutagenesis and kinetic experiments. On the basis of these findings, we suggest that Ser134 is the key residue that enables PST to discriminate PAP from AMP.
\end{abstract}

Sulfotransferases (STs) constitute a family of detoxicants that transfer a sulfuryl group from PAPS ${ }^{1}$ to an appropriate acceptor, such as phenol, alcohol, or amine (1) (Scheme 1). Many phenolic and alcoholic xenobiotics are substrates for one of the ST isoforms. Sulfonation of drugs and xenobiotics is associated with detoxification since biotransformation of a relatively hydrophobic compound into a more water soluble sulfuryl ester is more readily excreted $(1,2)$. Consequently, the biological functions of the ST-catalyzed sulfonation of endogenous substrates are continuing to be explored. For example, estrogen sulfonation is important in regulating estrogenic activity in estrogen responsive tissues $(3,4)$.

Sequence alignment of several STs reveals two highly conserved regions, one (PKSGTTW) in the N-terminal region and the other (RKGXXGDWKXXFT) in the C-terminal part $(5,6)$. Since only one natural sulfuryl donor (PAPS) has thus far been identified for all STs, it was suggested that these two highly conserved regions were involved in PAPS binding. The first X-ray crystal structure of a ST, mouse estrogen sulfotransferase (EST), confirmed that residues 259-265 and 45-51 of EST, indeed, interacted with PAPS

\footnotetext{
This research is supported by a grant from the National Science Council (89-2113-M-009-009 and 89-2311-B-009-010), Taiwan.

* To whom correspondence should be addressed: Department of Biological Science and Technology, National Chiao Tung University, 75 Po-Ai St., Hsinchu, Taiwan, ROC. Telephone: 886-3-5731983. Fax: 886-3-5729288. E-mail: ysyang@cc.nctu.edu.tw.

${ }^{1}$ Abbreviations: PAP, 3'-phosphoadenosine 5'-phosphate; AMP, adenosine 5'-monophosphate; PAPS, 3'-phosphoadenosine 5'-phosphosulfate; PST, phenol sulfotransferase; EST, estrogen sulfotransferase; pNP, $p$-nitrophenol.
}

Scheme 1: Sulfuryl Group Transfer in a Biological System

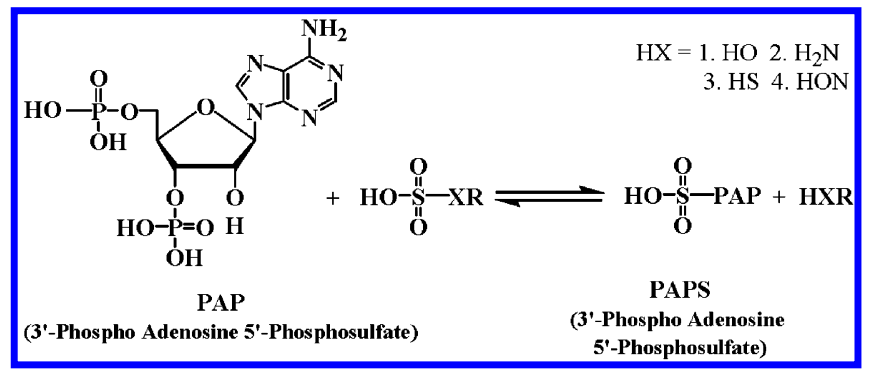

(7). Crystal structures of several ST isoforms have since been reported, which contributed important advances to our understanding of the reaction mechanism of these enzymes. They include the crystal structure of human dopamine/ catecholamine sulfotransferase $(8,9)$, the ST domain of human heparan sulfate $N$-deacetylase/ $N$-sulfotransferase 1 (10), and human hydroxysteroid sulfotransferase (11).

Several functional residues of sulfotransferase have been identified by site-directed mutagenesis. Notably, a highly conserved region, GxxGxxK, has been demonstrated to be important for PAPS binding with guinea pig EST (12). Lys59 and the phosphate binding loop of plant flavonol 3-ST were found to be critical for catalysis and nucleotide binding (13, 14). A chimeric construct of the human monoamine form (M-form) and the simple phenol form (P-form) of PST showed that the region encompassing residues 84-148 was the substrate specific/catalytic domain for both M-form and P-form PSTs. Two variable regions of M-form and P-form PSTs, residues 84-89 and 143-148, were also found to be 
responsible for their distinct enzymatic properties, especially residue 146 (Ala in P-form PST and Glu in M-form PST), which regulates the substrate specificity of M-form PST (15, 16). Mutagenesis studies of mouse EST revealed that Lys48 and His 108 were involved in the catalysis of the sulfuryl transfer reaction in EST (17) and Tyr81 contributed to EST substrate specificity (18). Also, mutagenesis studies of HNK-1 ST identified Lys128 and conserved residues (Arg189, Asp190, Pro191, and Ser197) as ones playing direct roles in substrate binding (19).

The nucleotide specificity of PST is much more diverse than previously anticipated, for many nucleotides other than PAP have recently been identified, which bind tightly to PST (20). Furthermore, two species of PST, $\alpha$ and $\beta$, have been isolated from one cDNA (21). In the presence of PAP, although both forms are equally active in carrying out the forward reaction shown in Scheme 1, the $\alpha$ form binds more tightly than the $\beta$ form to PAP. Also, only the $\beta$ form catalyzes the physiological transfer of PAPS to free phenol (the reverse reaction in Scheme 1). By exploiting the difference between the two forms of PST and focusing on AMP and PAP, we employed site-directed mutagenesis and molecular modeling methods to explore the molecular basis of the PST nucleotide specificity.

\section{EXPERIMENTAL PROCEDURES}

Materials. PfuTurbo DNA polymerase was purchased from Stratagene, and the DNA sequencing kit (BigDye Terminator Cycle Sequencing Ready Reaction) was obtained from Applied Biosystems. PAP and AMP were purchased from Sigma, and $p$-nitrophenyl sulfate (pNPS) was obtained from Fluka. We avoided using pNPS that contained $\sim 1 \%$ of total weight $p$-nitrophenol (pNP) as a contaminant. DEAESepharose was purchased from Pharmacia.

Molecular Modeling. Molecular coordinates of the mouse estrogen sulfotransferase (mEST) were used (PDB entry 1AQU) for carrying out molecular modeling on an Octane workstation using InsightII (Accelrys, San Diego, CA).

Site-Directed Mutagenesis of the cDNA Encoding PSTs. Using PfuTurbo DNA polymerase, site-directed mutagenesis experiments were performed with the method of QuickChange (Stratagene, La Jolla, CA). All primers for mutagenesis were purchased from Integrated DNA Technologies Inc. (Coralville, IA). Wild-type PST cDNA incorporated in the pET-3c expression vector (pET 3c11) was used as the template in conjunction with specific mutagenic primers (Table 1). Mutated cDNA sequences were confirmed with an ABI Prism 377 DNA sequencer (Applied Biosystems, Foster City, CA) following the standard protocol.

Expression and Purification of PST and Its Mutants. Overnight bacterial cultures were used to inoculate $5 \mathrm{~mL}$ of LB medium supplemented with ampicillin $(100-250 \mu \mathrm{g} /$ $\mathrm{mL}$ ). Cells, grown at $37{ }^{\circ} \mathrm{C}$ to $\mathrm{OD}_{600}$ of $0.3-0.5$, were transferred to $0.3 \mathrm{~L}$ of $\mathrm{LB}$ medium containing $50 \mu \mathrm{g} / \mathrm{mL}$ ampicillin. The cells were allowed to grow in shakers at 37 ${ }^{\circ} \mathrm{C}$ to $\mathrm{OD}_{600}$ of 5 , as measured by its $1: 10$ diluted culture. Overexpressed enzymes were extracted from bacteria by sonication and purified using a DEAE-Sepharose column as described previously (20). Some modifications of the PST expression and purification were necessary for optimization of the expression level and the purity of some mutants. For

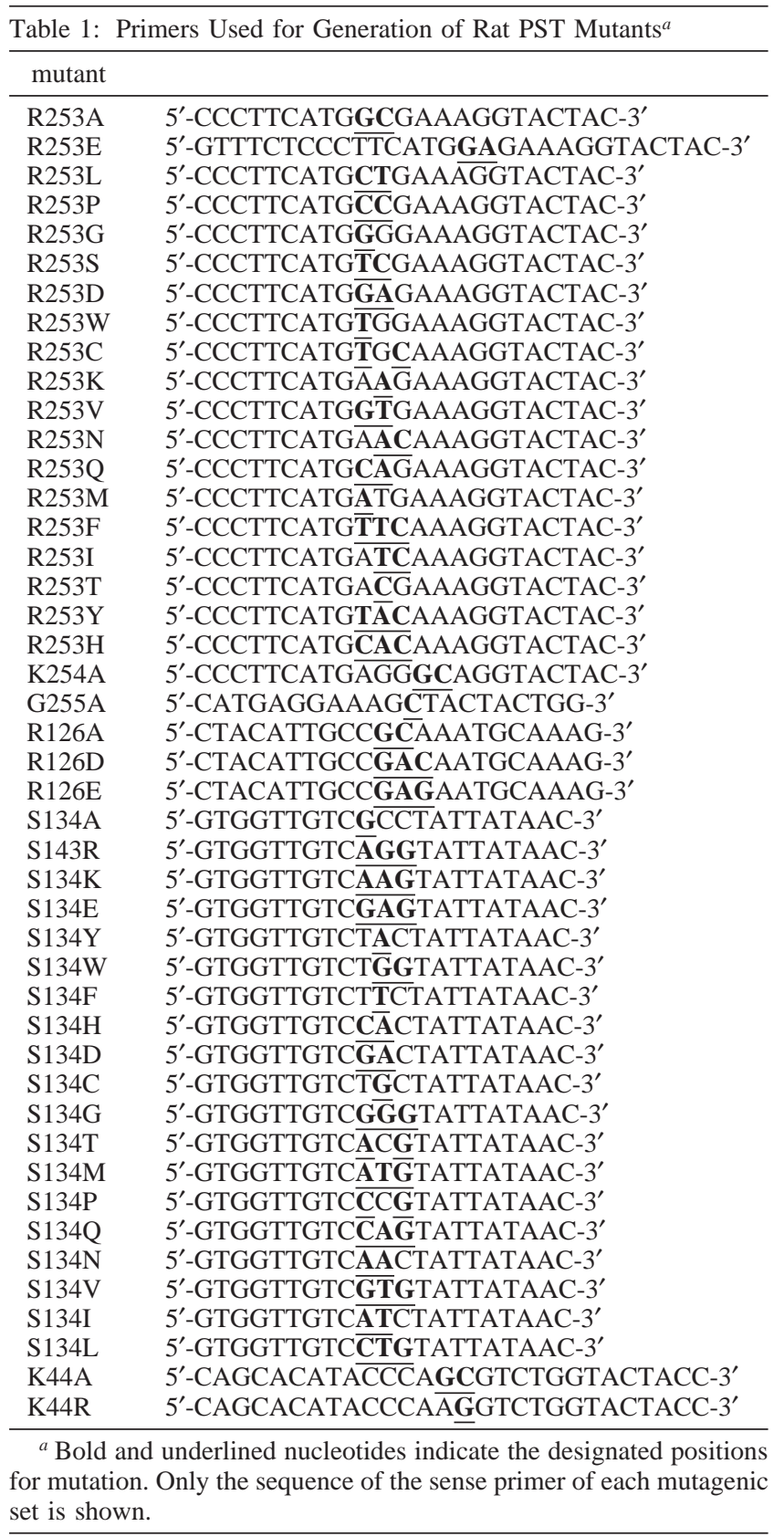

instance, to prevent the formation of inclusion bodies, some of mutants were expressed at $25^{\circ} \mathrm{C}$, and were purified at salt concentrations that were higher than that of the wild type.

Enzyme Assay. The PST assay was carried out via the formation of free $\mathrm{pNP}\left(\epsilon=10500 \mathrm{~cm}^{-1} \mathrm{M}^{-1}\right.$ at $\left.\mathrm{pH} 7.0\right)$. PAPS was generated first from pNPS and PAP as described previously $(20,21)$, and then used by the same enzyme for the sulfonation of 2-naphthol. The standard reaction mixture consisted of bis-tris propane $(100 \mathrm{mM})$ at $\mathrm{pH} 7.0,2$-mercaptoethanol (5 mM), pNPS (1 mM), 2-naphthol $(50 \mu \mathrm{M})$, and PAP $(2 \mu \mathrm{M})$. Sufficient enzyme was added for detection of an absorption change at $400 \mathrm{~nm}$. Enzymatic activity of the $\alpha$ form was determined in the absence of PAP as described previously $(20,21)$. The reaction was carried out for 2-5 min in cuvettes with path lengths of $1 \mathrm{~cm}$ at $25^{\circ} \mathrm{C}$ in a Hitachi U3300 spectrophotometer. Under these conditions, the reaction was linear with respect to time and enzyme concentration. A unit of activity represents the amount of 


\begin{tabular}{|c|c|c|c|c|c|c|c|}
\hline $\begin{array}{r}\text { rPST } \\
\text { mEST } \\
\text { SULT1A3 } \\
\text { hHST } \\
\text { F3ST }\end{array}$ & 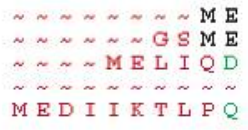 & 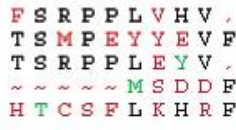 & 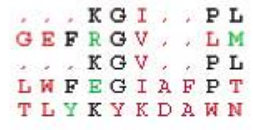 & 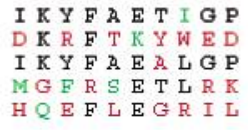 & 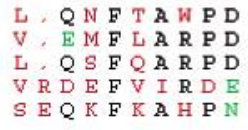 & 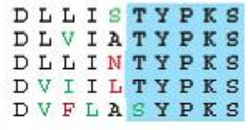 & $\begin{array}{l}45 \\
51 \\
49 \\
45 \\
60\end{array}$ \\
\hline $\begin{array}{r}\text { rPST } \\
\text { mEST } \\
\text { SULT1A3 } \\
\text { hHST } \\
\text { F3ST }\end{array}$ & 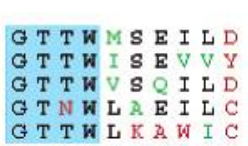 & 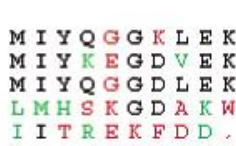 & 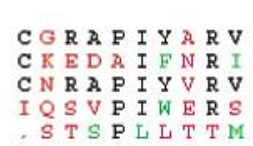 & $\begin{array}{l}\text { P F L E E K C P G V } \\
\text { P Y L E C R N E D L } \\
\text { P F L E V N D P G E } \\
\text { P W V E S E } \\
\text { P H. D C. I P L L }\end{array}$ & $\begin{array}{l}\text { P S G L E T L E E T } \\
\text { ING I K K Q L K E K } \\
\text { P S G L E T L K D T } \\
\text { I G Y T T R L S E T } \\
\text { E K D L E E K I Q E E N }\end{array}$ & 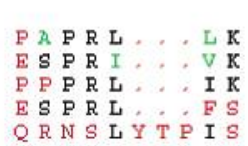 & $\begin{array}{l}102 \\
108 \\
106 \\
97 \\
116\end{array}$ \\
\hline $\begin{array}{r}\text { rPST } \\
\text { mEST } \\
\text { SULT1A3 } \\
\text { hHST } \\
\text { F3ST }\end{array}$ & 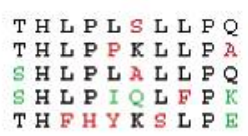 & 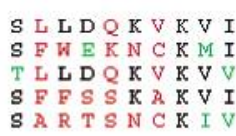 & 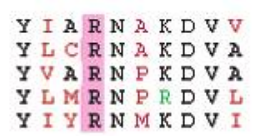 & 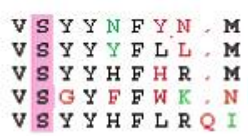 & 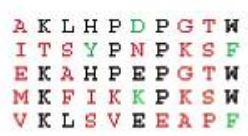 & $\begin{array}{l}D S F L E N F M D G \\
S E F V E K F M Q G \\
D S F C E K F M A G \\
E \text { E Y F E W FCQG } \\
E \text { E A F D E F C Q }\end{array}$ & $\begin{array}{l}161 \\
167 \\
165 \\
156 \\
176\end{array}$ \\
\hline $\begin{array}{r}\text { rPST } \\
\text { mEST } \\
\text { SULT1A3 } \\
\text { hHST } \\
\text { F3ST }\end{array}$ & 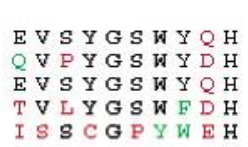 & 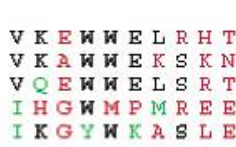 & $\begin{array}{llll}\text { H P V L Y L F } & \text { Y } \\
\text { S R V L F M F } & Y \\
\text { H P V L Y L F } & Y \\
\text { KN F L L L S } & \text { Y } \\
\text { K P E I E L E L K Y }\end{array}$ & 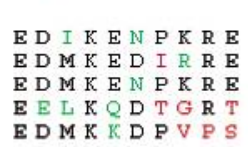 & $\begin{array}{lllllllll}I & K & K & I & L & E & F & L & G\end{array}$ & $\begin{array}{l}\text { SLPEET } \\
\text { K P SAEL } \\
\text { SLPEET } \\
\text { TLEEPEE } \\
\text { PETPKEEEAG }\end{array}$ & $\begin{array}{l}215 \\
221 \\
219 \\
210 \\
236\end{array}$ \\
\hline $\begin{array}{r}\text { rPST } \\
\text { mEST } \\
\text { SULTIA3 } \\
\text { hHST } \\
\text { F3ST }\end{array}$ & 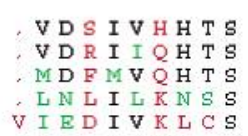 & $\begin{array}{l}F K K M K E N C M T \\
F Q E M K N N P S T \\
F K E M K K N P M T \\
F Q S M K E N K M S \\
F E K L S S L E V N\end{array}$ & 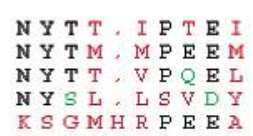 & 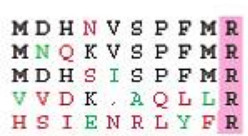 & 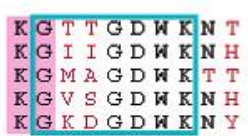 & 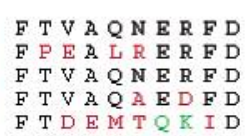 & $\begin{array}{l}273 \\
279 \\
277 \\
267 \\
296\end{array}$ \\
\hline $\begin{array}{r}\text { rPST } \\
\text { mEST } \\
\text { SULT1A3 } \\
\text { hHST } \\
\text { F3ST }\end{array}$ & 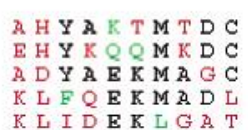 & $\begin{array}{l}\text { TVK K F R MEL } \\
\text { SLS FR S E L } \\
\text { PRE E F P W E } \\
\text { G L V V K } \sim \sim \sim \sim\end{array}$ & & & & & \\
\hline
\end{tabular}

FIGURE 1: Sequence alignment of rat phenol sulfotransferase (rPST) and its homologues from mice, humans, and plants: mouse estrogen sulfotransferase (mEST), human dopamine/catecholamine sulfotransferase (SULT1A3), human hydroxysteroid sulfotransferase (hHST), and plant flanvol 3-sulfotransferase (F3ST). The PSB loop that interacts with the $5^{\prime}$-phosphate of PAP is shown with a blue background. Residues involved in binding the 3'-phosphate of PAP are shown with a magenta background. The conserved GxxGxxK sequence motif is enclosed by a teal box. The alignment was generated using Pileup and Prettybox programs in GCG.

enzyme required to catalyze the formation of $1 \mu \mathrm{mol}$ of pNP/ min in the standard assay. Specific activity is given in units per milligram of protein.

Nucleotides as Cofactors of PST. Various concentrations of PAP or AMP were incubated with pNPS $(1 \mathrm{mM})$, mercaptoethanol $(5 \mathrm{mM}), 2$-naphthol $(50 \mu \mathrm{M})$, and bis-tris propane $(100 \mathrm{mM})$ at $\mathrm{pH} 7.0$ followed by addition of PST. The enzymatic activity was determined by the change in absorbance of the generated pNP at $400 \mathrm{~nm}$. At least three measurements were collected at each nucleotide concentration. The apparent kinetic constants for PAP and AMP were obtained using a nonlinear regression method implemented in GraFit data analysis software (Erithacus Software Ltd.). Nucleotide concentrations were determined at $260 \mathrm{~nm}$ using $\epsilon$ values of 15100 and $15400 \mathrm{M}^{-1} \mathrm{~cm}^{-1}$ for PAP and AMP, respectively.

\section{RESULTS}

Identification of Residues Involved in PAP Binding Using Sequence Alignment and Structural Modeling. Sequence alignment of the rat PST (rPST), several cytosolic STs, and a membrane-anchored ST reveals that these proteins are highly homologous (Figure 1); notably, residues that are directly implicated in the catalytic mechanism and substrate specificity are conserved. Since PAP, unlike AMP, contains a phosphate group at the $3^{\prime}$-position of the ribose ring, we examined the position of residues in the region close to the PAP binding site in our PST model for the possible cause of the change in the nucleotide specificity of PST (Figure 2A). Inspection of the PST model shows that five residues (Arg253, Lys254, Gly255, Arg126, and Ser134) contribute to the formation of the 3 '-phosphate binding pocket (Figure
2B). Whereas backbone $\mathrm{NH}$ groups of residues $253-255$ interact with the 3'-phosphate of PAP, only side chains of Ser134 and Arg126 hydrogen bond with the $3^{\prime}$-phosphate of PAP. Residues 253-255 precede the conserved sequence motif, ${ }^{255} \mathrm{GxxGxxK}{ }^{261}$, which has been shown to play a role in PAPS binding (12). In contrast, Arg126 precedes a conserved helix and Ser134 is a constituent of the same helix (7). Although both $\mathrm{O}_{\gamma}$ of Ser134 and the guanidinium group of Arg126 hydrogen bond to the 3'-phosphate of PAP, it is the latter that effectively balances the net charge around the phosphate (see below).

The other conserved region, ${ }^{41}$ TYPKSGTTW ${ }^{49}$, known as the phosphate-sulfate binding loop (PSB loop), interacts with PAP, of which Lys44, Ser45, Gly46, and Thr47 interact with the 5'-phosphate. Accordingly, we have selected Arg253, Lys254, Gly255, Arg126, Ser134, and Lys44 as targets of mutagenesis studies in an effort to identify a residue(s) that enables PST to distinguish PAP from AMP.

Site-Directed Mutagenesis of Selected Residues and Preparation of PST Mutants. When sequences of the selected PST mutants (Table 1) were verified by DNA sequencing, they were expressed in Escherichia coli. The homogenate of the cell extract was used to determine the total enzymatic activity, the population ratio of the two forms of the enzyme (Table 2), and $K_{\mathrm{m}}$ (Table 3). Selected PST mutants were purified to homogeneity as confirmed by SDS-polyacrylamide gel electrophoresis (data not shown) followed by determination of their $K_{\mathrm{m}}$ and $k_{\mathrm{cat}}$ (Table 4).

Point Mutations of PST Affect Formation of Two Enzymatic Forms. Two forms of PST, $\alpha$ and $\beta$, are obtained when the recombinant PST is expressed. If the K254A and K44R mutants are excluded, almost all mutants are of the $\beta$ form 


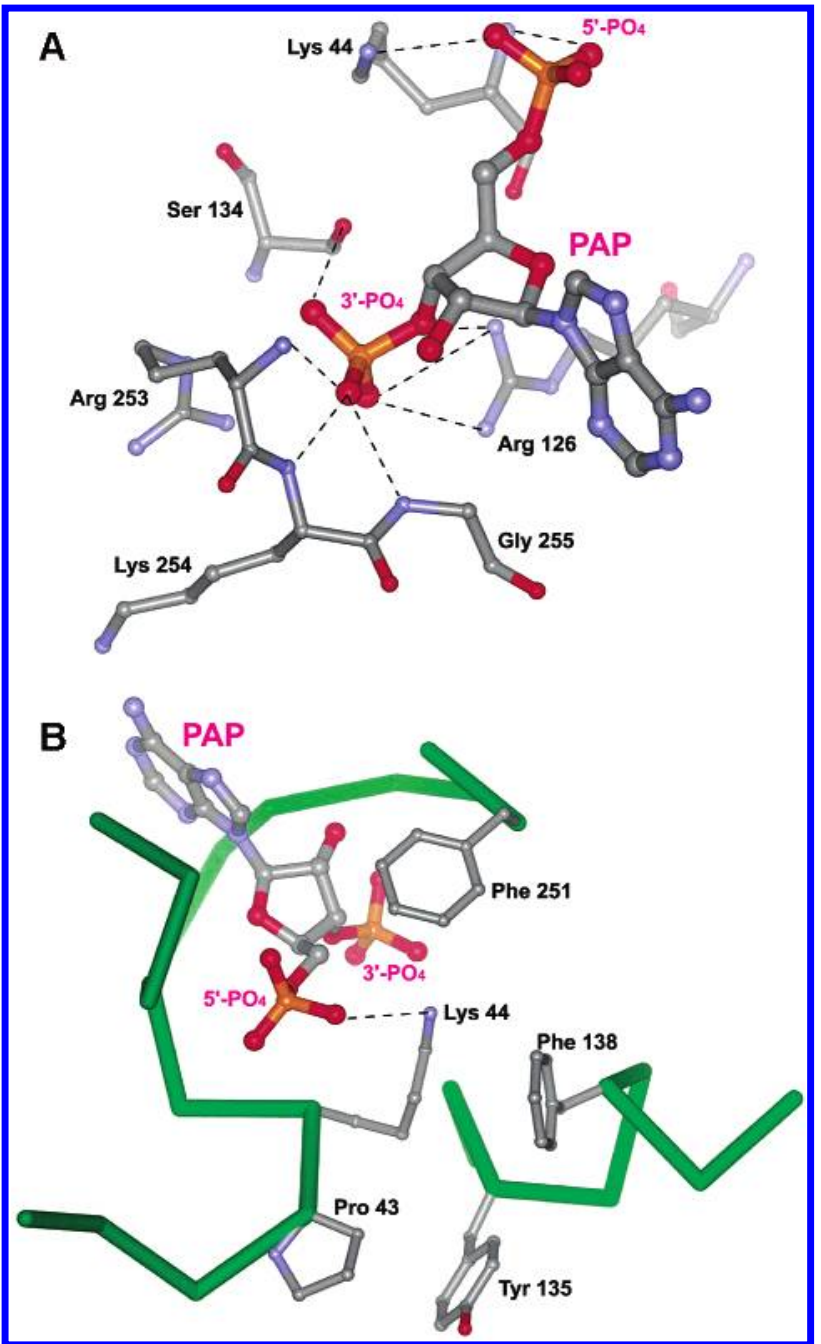

FIGURE 2: Molecular modeling of rPST. The PST model was built using the coordinates of the crystal structure of the mouse estrogen ST. (A) Nucleotide binding site of PAP. Selected polar interactions (black dashed lines) between PAP and the PST residues are represented as ball-and-stick models. Side chains of Arg126 and Ser134 directly interact with the $3^{\prime}$-phosphate of PAP. In contrast, only the NH backbone of Arg253, Lys254, and Gly255 hydrogen bonds with the 3'-phosphate of PAP. Carbon, nitrogen, oxygen, and phosphorus atoms are shown in gray, blue, red, and orange, respectively. (B) Ball-and-stick representation of PAP, Lys44, and the hydrophobic residues surrounding Lys44. Only side chains of the PST residues are shown, and the green portion represents the $\mathrm{C} \alpha$ backbone. This figure was produced with WebLab Viewer Lite (Accelrys).

(Table 2), confirming the notion that four residues (Arg253, Gly255, Arg126, and Ser134) are important for the binding of PAP, which is consistent with the X-ray crystal structure of mEST (7) from which the PST model was derived. Approximately $30 \%$ of the K254A mutant population is of the $\alpha$ form, which is similar to that of the wild type, implying that the PAP interaction with Lys254 may not be as important as those of Arg253 and Gly255.

Mutation of Lys44 leads to the formation of a different form of the enzyme, depending on what residue is replaced (Table 2). Whereas the mutation of Lys44 to alanine results in the formation of exclusively the $\beta$ form, that of Lys 44 to arginine predominantly produces the $\alpha$ form $(\sim 88 \%)$. Lys 44 resides at the center of a hydrophobic pocket consisting of Pro43, Tyr135, Phe138, and Phe251, with which it makes weak hydrophobic interactions (Figure 2B). Additionally, the

\begin{tabular}{|c|c|c|c|c|c|}
\hline enzyme & $\begin{array}{c}\alpha \text { form } \\
\text { activity } \\
(\%)\end{array}$ & $\begin{array}{l}\beta \text { form } \\
\text { activity } \\
(\%)\end{array}$ & enzyme & $\begin{array}{c}\alpha \text { form } \\
\text { activity } \\
(\%)\end{array}$ & $\begin{array}{c}\beta \text { form } \\
\text { activity } \\
(\%)\end{array}$ \\
\hline wild type & 26 & 74 & G255A & 0 & 100 \\
\hline R253A & 0 & 100 & R126A & 0 & 100 \\
\hline $\mathrm{R} 253 \mathrm{E}$ & 0 & 100 & R126D & $\mathrm{ND}^{c}$ & $\mathrm{ND}^{c}$ \\
\hline R253L & $0^{b}$ & $100^{b}$ & R126E & $\mathrm{ND}^{c}$ & $\mathrm{ND}^{c}$ \\
\hline $\mathrm{R} 253 \mathrm{P}$ & $9^{b}$ & $91^{b}$ & S134A & 0 & 100 \\
\hline R253G & $0.6^{b}$ & $99.4^{b}$ & S134R & 0 & 100 \\
\hline R253S & $\mathrm{ND}^{c}$ & $\mathrm{ND}^{c}$ & S134K & 0 & 100 \\
\hline R253D & $\mathrm{ND}^{c}$ & $\mathrm{ND}^{c}$ & S134E & 0 & 100 \\
\hline R253W & $1^{b}$ & $99^{b}$ & S134Y & $\mathrm{ND}^{c}$ & $\mathrm{ND}^{c}$ \\
\hline R253C & $1.5^{b}$ & $98.5^{b}$ & S134W & $\mathrm{ND}^{c}$ & $\mathrm{ND}^{c}$ \\
\hline R253K & 0 & 100 & S134F & $\mathrm{ND}^{c}$ & $\mathrm{ND}^{c}$ \\
\hline R253V & $\mathrm{ND}^{c}$ & $\mathrm{ND}^{c}$ & S134H & 0 & 100 \\
\hline R253N & $3^{b}$ & $97^{b}$ & S134D & 0 & 100 \\
\hline R253Q & $0.8^{b}$ & $99.2^{b}$ & S134C & 0 & 100 \\
\hline R253M & $0.9^{b}$ & $99.1^{b}$ & S134G & 0 & 100 \\
\hline R253F & $\mathrm{ND}^{c}$ & $\mathrm{ND}^{c}$ & S134T & 0 & 100 \\
\hline R253I & $\mathrm{ND}^{c}$ & $\mathrm{ND}^{c}$ & S134M & $\mathrm{ND}^{c}$ & $\mathrm{ND}^{c}$ \\
\hline R253T & $0.9^{b}$ & $99.1^{b}$ & S134P & $\mathrm{ND}^{c}$ & $\mathrm{ND}^{c}$ \\
\hline R253Y & $\mathrm{ND}^{c}$ & $\mathrm{ND}^{c}$ & S134Q & 0 & 100 \\
\hline $\mathrm{R} 253 \mathrm{H}$ & $\mathrm{ND}^{c}$ & $\mathrm{ND}^{c}$ & S134N & 0 & 100 \\
\hline K254A & 29 & 71 & S134V & $\mathrm{ND}^{c}$ & $\mathrm{ND}^{c}$ \\
\hline K44A & 0 & 100 & S134I & $\mathrm{ND}^{c}$ & $\mathrm{ND}^{c}$ \\
\hline K44R & 88 & 12 & S134L & $\mathrm{ND}^{c}$ & $\mathrm{ND}^{c}$ \\
\hline
\end{tabular}

${ }^{a}$ The percent population of the PAP-bound PST, $\alpha$, was determined by the standard assay in the presence and absence of PAP, which gives the total enzyme activity and the activity of $\alpha$, respectively $(22,23)$. ${ }^{b}$ Obtained using the enzyme crude extract. ${ }^{c}$ Not determined due to undetectable enzymatic activity.

Table 3: Effect of Mutation on the $K_{\mathrm{m}}$ of PAP and AMP for Residues that Provide Backbone Interactions to PAP

\begin{tabular}{lccccc}
\hline & \multicolumn{2}{c}{ PAP } & & \multicolumn{2}{c}{ AMP } \\
\cline { 2 - 3 } \cline { 5 - 6 } enzyme & $K_{\mathrm{m}}(\mu \mathrm{M})$ & $x$-fold & & $K_{\mathrm{m}}(\mu \mathrm{M})$ & $x$-fold \\
\hline wild type & $0.04 \pm 0.01$ & 1 & $430 \pm 11$ & 1 \\
R253A & $2.3 \pm 0.1$ & 58 & & $2200 \pm 240$ & 5 \\
R253E & $130 \pm 6$ & 3700 & & $3300 \pm 400$ & 8 \\
R253L & $3 \pm 0.2^{a}$ & 75 & & $1500 \pm 230^{a}$ & 3.6 \\
R253P & $34.3 \pm 1.6^{a}$ & 860 & & $2700 \pm 300^{a}$ & 6.5 \\
R253G & $1.8 \pm 0.5^{a}$ & 45 & & $2300 \pm 370^{a}$ & 5.5 \\
R253W & $2.3 \pm 0.2^{a}$ & 57 & & $1500 \pm 570^{a}$ & 3.5 \\
R253C & $0.8 \pm 0.25^{a}$ & 20 & & $3100 \pm 1200^{a}$ & 7.5 \\
R253V & $0.9 \pm 0.3^{a}$ & 22 & & $2300 \pm 130^{a}$ & 5.5 \\
R253N & $0.34 \pm 0.06^{a}$ & 8.5 & $2800 \pm 800^{a}$ & 6.5 \\
R253Q & $2 \pm 1^{a}$ & 50 & & $1700 \pm 100^{a}$ & 4 \\
R253M & $2.3 \pm 0.7^{a}$ & 57 & & $4200 \pm 760^{a}$ & 10 \\
R253I & $3.4 \pm 1.4^{a}$ & 85 & & $2500 \pm 200^{a}$ & 6 \\
R253T & $13 \pm 3^{a}$ & 340 & & $4040 \pm 450^{a}$ & 10 \\
K254A & $0.03 \pm 0.004$ & 0.7 & & $230 \pm 30$ & 0.5 \\
G255A & $1.4 \pm 0.3$ & 35 & $1000 \pm 150$ & 2.5 \\
\hline
\end{tabular}

${ }^{a}$ Obtained using the enzyme crude extract.

side chain of Lys44 donates one strong hydrogen bond to the 5'-phosphate of PAP (Figure 2A). Whereas mutation of Lys44 to Ala inhibits this electrostatic interaction, an Arg substitution of Lys44 reinforces it. The model suggests that the guanidinium moiety of Arg, apart from possibly forming two hydrogen bonds with the $5^{\prime}$-phosphate of PAP, may make $\pi-\pi$ interactions with the side chain of Phe138 (Figure 2B).

Mutation of Amino Acids that Provide Backbone Interactions with PAP Affects the Binding Affinity of both PAP and $A M P$. Factors influencing $K_{\mathrm{m}}, k_{\mathrm{cat}}$ and the catalytic efficiency of the enzyme by point mutation of Arg253, Lys254, and Gly255 were analyzed (Tables 3 and 4). 
Table 4: Catalytic Efficiency of PST and Its Mutants Using PAP and $\mathrm{AMP}^{a}$

\begin{tabular}{|c|c|c|c|c|c|c|c|}
\hline \multirow[b]{2}{*}{ enzyme } & \multicolumn{3}{|c|}{ PAP } & \multicolumn{3}{|c|}{ AMP } & \multirow[b]{2}{*}{$\begin{array}{l}\text { specificity ratio }^{b} \\
\text { (PAP/AMP) }\end{array}$} \\
\hline & $K_{\mathrm{m}}(\mu \mathrm{M})$ & $k_{\text {cat }}\left(\min ^{-1}\right)$ & $\begin{array}{c}k_{\mathrm{cat}} / K_{\mathrm{m}} \\
\left(\mathrm{min}^{-1} / \mu \mathrm{M}\right)\end{array}$ & $K_{\mathrm{m}}(\mu \mathrm{M})$ & $k_{\text {cat }}\left(\min ^{-1}\right)$ & $\begin{array}{c}k_{\text {cat }} / K_{\mathrm{m}} \\
\left(\mathrm{min}^{-1} / \mu \mathrm{M}\right)\end{array}$ & \\
\hline wild type & $0.04 \pm 0.01$ & $59 \pm 2$ & 1475 & $430 \pm 110$ & $56 \pm 4$ & 0.13 & 11300 \\
\hline R253A & $2.3 \pm 0.1$ & $63 \pm 0.3$ & 27 & $2200 \pm 240$ & $31 \pm 1$ & 0.014 & 1900 \\
\hline R253E & $130 \pm 6$ & $62 \pm 0.7$ & 0.48 & $3300 \pm 430$ & $37 \pm 1$ & 0.01 & 48 \\
\hline K254A & $0.03 \pm 0.004$ & $57 \pm 1$ & 1900 & $230 \pm 30$ & $55 \pm 1$ & 0.24 & 7900 \\
\hline G255A & $1.4 \pm 0.3$ & $20 \pm 0.5$ & 14 & $1000 \pm 150$ & $10 \pm 0.5$ & $9.6 \times 10^{-3}$ & 1500 \\
\hline $\mathrm{R} 126 \mathrm{~A}$ & $3600 \pm 700$ & $2.6 \pm 0.2$ & $7.2 \times 10^{-4}$ & $5000 \pm 800$ & $0.31 \pm 0.01$ & $6.2 \times 10^{-5}$ & 12 \\
\hline S134A & $0.17 \pm 0.03$ & $39 \pm 1$ & 229 & $800 \pm 70$ & $20 \pm 1$ & 0.025 & 9200 \\
\hline S134R & $1100 \pm 130$ & $3 \pm 0.1$ & $2.8 \times 10^{-3}$ & $430 \pm 40$ & $2.3 \pm 0.04$ & $5.3 \times 10^{-3}$ & 0.5 \\
\hline S134K & $240 \pm 20$ & $2.5 \pm 0.05$ & 0.01 & $2100 \pm 270$ & $0.45 \pm 0.02$ & $2.1 \times 10^{-4}$ & 48 \\
\hline S134E & $4000 \pm 170$ & $23.1 \pm 0.3$ & $5.8 \times 10^{-3}$ & $470 \pm 70$ & $27 \pm 1$ & 0.06 & 0.1 \\
\hline S134D & $1300 \pm 160$ & $18 \pm 1$ & 0.014 & $2000 \pm 750$ & $26 \pm 3$ & 0.013 & 1.1 \\
\hline S134C & $1.8 \pm 0.6$ & $18 \pm 1$ & 10 & $4000 \pm 2400$ & $18 \pm 4$ & $4.5 \times 10^{-3}$ & 2200 \\
\hline S134G & $0.8 \pm 0.3$ & $42 \pm 2$ & 53 & $1800 \pm 320$ & $15 \pm 1$ & $8.3 \times 10^{-3}$ & 6400 \\
\hline S134H & $\gg 10000^{c}$ & $\mathrm{ND}^{c}$ & - & $3700 \pm 370$ & $1.3 \pm 0.04$ & $3.5 \times 10^{-4}$ & $<1$ \\
\hline S134Q & $1200 \pm 20$ & $1.2 \pm 0.2$ & $1 \times 10^{-3}$ & $3500 \pm 340$ & $6.2 \pm 0.2$ & $1.8 \times 10^{-3}$ & 0.6 \\
\hline S134N & $590 \pm 60$ & $21 \pm 1$ & 0.04 & $4100 \pm 480$ & $8 \pm 0.2$ & $2 \times 10^{-3}$ & 20 \\
\hline $\mathrm{S} 134 \mathrm{~T}$ & $10 \pm 2$ & $17 \pm 1$ & 1.7 & $3000 \pm 270$ & $8 \pm 0.2$ & $2.7 \times 10^{-3}$ & 630 \\
\hline K44A & $26 \pm 2$ & $0.8 \pm 0.01$ & 0.03 & $12200 \pm 1000$ & $2 \pm 0.1$ & $1.6 \times 10^{-4}$ & 190 \\
\hline
\end{tabular}

${ }^{a}$ Assay conditions are described in Experimental Procedures. ${ }^{b}$ The specificity ratio (PAP/AMP) is calculated as $\left(k_{\text {cat }} / K_{\mathrm{m}}\right)_{\mathrm{PAP}} /\left(k_{\text {cat }} / K_{\mathrm{m}}\right)_{\mathrm{AMP}}{ }^{c} K_{\mathrm{m}}$ is too high to be determined accurately. PST activity increases almost linearly even when $7.9 \mathrm{mM}$ PAP is used.

Mutation of Arg253 to Ala, Glu, Leu, Pro, Gly, Trp, Cys, Val, Asn, Gln, Met, Ile, or Thr increases $K_{\mathrm{m}(\mathrm{PAP})}$ from 20 -

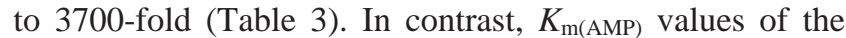
same mutants are 3-10 times higher than that of the wild type. Although, compared to that of the wild type, R253A and R253E mutants, respectively, exhibit $10^{2}$ - and $10^{4}$-fold decreases in catalytic efficiency $\left(k_{\text {cat }} / K_{\mathrm{m}}\right)$ of PAP, they display an $\sim 10$ times lower $k_{\text {cat }} / K_{\mathrm{m}}$ for AMP (Table 4 ). The specificity ratios (PAP/AMP) of R253A and R253E were 1900 and 48 , respectively, which are much lower than that of the wild type (11 300). Since the $k_{\text {cat }}$ values (using either PAP or AMP) of these two mutants remain relatively constant, $K_{\mathrm{m}}$ is then responsible for the observed variation (Table 4). According to the PST model, the backbone NH group of Arg253 makes one hydrogen bond with the 3 '-phosphate of PAP, while its side chain is exposed to the solvent and does not directly interact with the phosphate (Figure 2A). Thus, mutation of Arg253 to either Ala or Glu cannot have a significant effect on the $k_{\text {cat }}$ of either PAP or AMP.

The mutational effect at Gly255 is similar to that of Arg253 (Tables 2-4), for G255A displays 100 and 10 times lower $k_{\text {cat }} / K_{\mathrm{m}}$ values for PAP and AMP, respectively, than the wild type. K254A is the only mutant that behaves like the wild type. Mutation of Lys 254 to a small hydrophobic residue such as Ala does not alter the enzyme specificity and activity (Table 4). Consistent with the impact of mutation on the formation of two forms of the enzyme, these results indicate that Lys254, residing between Arg253 and Gly255, is especially stable in the overall conformation.

Mutational Analysis of Lys44. The side chain of Lys44 hydrogen bonds to the $5^{\prime}$-phosphate of PAP (Figure 2A). Structural and functional analysis of mEST and flavonol 3-ST suggested that the corresponding lysine was involved in the catalysis of the sulfuryl transfer reaction $(14,17)$. Mutation of Lys44 to Arg enhances the affinity of PST for PAP, for the mutant population mainly consists of the $\alpha$ form (Table 2 ). In contrast, the K44A mutant exhibits an increased $K_{\mathrm{m}}$ and a decreased $k_{\text {cat }}$ for both PAP and AMP. As expected, the $K_{\mathrm{m} \text { (AMP) }}$ of $\mathrm{K} 44 \mathrm{~A}$ is the highest among all the mutants reported here (Tables 3 and 4), for the mutation of Lys44 offers no advantage for PST with respect to its binding of AMP. Lys44 has been proposed to be an essential residue not only in the binding of PAP but also in catalysis (13, 17). In our study, this residue is shown to be more important for nucleotide binding than catalysis, for the ratios of $K_{\mathrm{m} \text { (PAP) }}$ and $K_{\mathrm{m} \text { (AMP) }}$ of K44A to those of the wild type are 660 and 29 , respectively, but the ratios of $k_{\text {cat(PAP) }}$ and $k_{\text {cat(AMP) }}$ of K44A to those of the wild type are 76 and 27, respectively.

Affinity of PST for PAP but Not for AMP Strongly Depends on the Type of Residue Replacing either Arg126 or Ser134. Side chains of Arg126 and Ser134 directly interact with the 3 -phosphate of PAP (Figure 2A). Mutation of Arg126 to Ala increases $K_{\mathrm{m} \text { (PAP) }}$ and $K_{\mathrm{m} \text { (AMP) }}$ 90000- and 12-fold, respectively (Table 4). The guanidinium of Arg126 forms a $\pi-\pi$ interaction the aromatic group of Tyr 189 , the $\mathrm{OH}$ group of which hydrogen bonds to the adenine (Figure 3A). Mutation of the arginine, therefore, directly affects binding of AMP and PAP, for it destabilizes Tyr189.

By comparison, the $K_{\mathrm{m}(\mathrm{PAP})}$ of the mutant enzyme increases up to $>250000$-fold compared to that of the wild type, depending on the residue to which Ser134 was mutated, even though $K_{\mathrm{m}(\mathrm{AMP})}$ increases nearly 10 -fold or remains unchanged (Table 4). Since replacement of PAP with AMP lacking the 3 -phosphate opens up a space originally reserved for the phosphate (Figure 3A), mutating Ser134 to a bulky residue may significantly affect the affinity of PAP but not AMP for PST. This is evident by our systematic mutation of Ser134 to other amino acids (Table 4). Substitution of Ser134 with a bulky residue has reversed the nucleotide preference of PST from PAP to AMP. For instance, see the data for S134R, S134H, S134E, and S134Q mutants in Table 4. The specificity ratio (Table 4 ) of these mutants to the wild type decreases $\sim 24000-146000$-fold. Of the four aforementioned mutants, the $K_{\mathrm{m} \text { (AMP) }}$ values of S134R and S134E remain the same compared with that of the wild type. However, the $K_{\mathrm{m} \text { (AMP) }}$ values of these mutants are smaller than their $K_{\mathrm{m}(\mathrm{PAP})}$ values. Mutation of Ser134 to a basic 


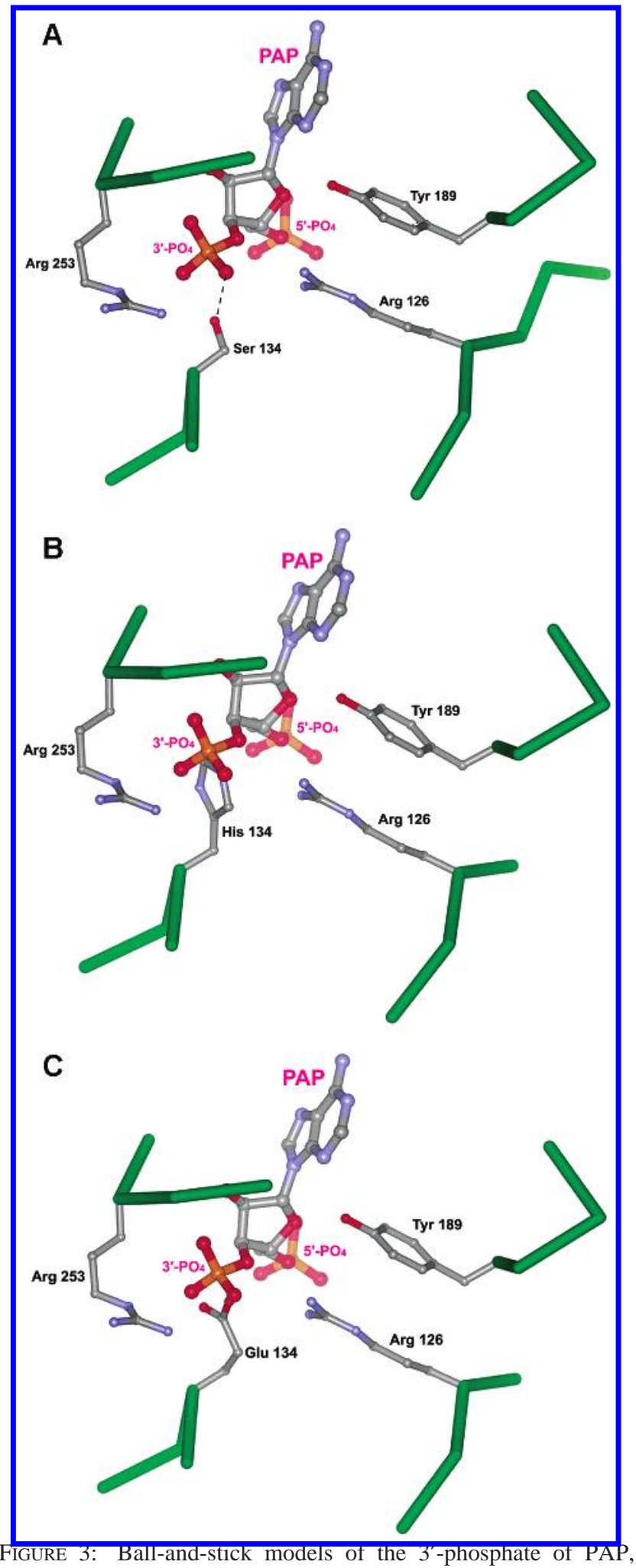

Ser134, and its mutants. The interactions of wild-type PST with PAP and AMP, which lacks the 3'-phosphate, are compared with those of the S134E and S134H mutants. (A) Wild-type PST via its designated residues properly interacts with the 3'-phosphate of PAP. (B) The mutant $\mathrm{S} 134 \mathrm{H}$ occupies the presumed location of the 3'phosphate. The mutant introduces additional unfavorable positive charges between Arg126 and Arg253. (C) The S134E mutant, although like S134E inhibits PAP binding, its negatively charged side chain, favorably interacts with both Arg126 and Arg253. This figure was produced with WebLab Viewer Lite (Accelrys).

residue decreases both $k_{\text {cat(PAP) }}$ and $k_{\text {cat(AMP) }}(\mathrm{S} 134 \mathrm{R}, \mathrm{S} 134 \mathrm{~K}$, and $\mathrm{S} 134 \mathrm{H})$, while that of an acidic residue does not (S134E and S134D). These findings imply that the local electrostatic interactions play critical roles in the activity of these mutant enzymes but not in their specificity. In fact, the PST model provides important clues about how these mutations affect the nucleotide binding of the enzyme. Ser134 is located between Arg126 and Arg253 (Figure 3A). Replacing Ser134 with a basic residue having a bulky side chain such as His or Glu occupies the presumed position of the 3'-phosphate of PAP, thereby impeding PAP binding. Since the positively charged side chain of His replaces the negatively charged 3'-phosphate residing between Arg126 and Arg253, its presence introduces unfavorable charge-charge interactions (Figure 3B). In contrast, the Ser134 to Glu mutation provides a favorable electrostatic environment, for the side chain of Glu can interact with guanidinium groups of Arg126 and Arg253. As a result, the S134E mutant has the lowest specificity ratio, $K_{\mathrm{m}(\mathrm{AMP})}$ being lower than $K_{\mathrm{m}(\mathrm{PAP})}$, and has $k_{\text {cat(PAP) }}$ and $k_{\text {cat(AMP) }}$ values similar to those of the wild type.

Since the mutation of both Lys44 and Ser134 has shown to be highly effective, comparative mutagenesis studies of Lys44 and Ser134 can thus be utilized in studying the mechanism of the interaction of PST with AMP and PAP.

\section{DISCUSSION}

The major goal of this research was to identify a residue(s) that had a critical role in enabling PST to distinguish PAP from AMP. Among the residues selected for mutagenesis studies, only Ser134 of PST appears to regulate its nucleotide specificity between AMP and PAP. Of the four mutants (S134E, S134R, S134Q, and S134H) which have a specific preference for binding AMP rather than PAP, the S134E mutant exhibits the highest specificity $(>100000$ folds) compared to that of the wild type. As summarized in Figure 4A, substituting Ser134 with a bulky residue dramatically increases $K_{\mathrm{m}(\mathrm{PAP})}$, but not $K_{\mathrm{m} \text { (AMP) }}$. The $K_{\mathrm{m}(\mathrm{AMP})}$ values of S134E, S134R, and S134H are even lower than $K_{\mathrm{m} \text { (PAP). }}$ Also, $k_{\text {cat(PAP) }}$ and $k_{\text {cat(AMP) }}$ are affected by the size of a side chain as a larger size reduces $k_{\text {cat }}$ (Figure 4B). Whereas substitutions of Ser134 to acidic residues, Glu and Asp, show that the mutants have $k_{\text {cat }}$ values comparable to that of the wild type, both S134K and S134R have significantly lower $k_{\text {cat }}$ values (Figure 4B). Consequently, mutation of Ser134 to a bulky residue tends to decrease the catalytic efficiency of PST toward PAP but not AMP (Figure 4C).

Mutation of Ser134 to either His or Glu causes a dramatic change in the PAP catalytic efficiency $\left[k_{c a l} / K_{\mathrm{m}(\mathrm{PAP})}\right]$ (Figure 4C), which results in inhibition of PAP binding. However, the kinetic data imply two different scenarios. The S134H mutant, by introducing an extra positive charge, unbalances the net charge of the active site (Figure 3B), while the S134E mutant reinforces it (Figure 3C). The side chain of Glu134, by occupying the presumed location of the 3 -phosphate of PAP, contributes negative charge that is comparable to that of the phosphate. This assumption is further confirmed by the fact that the S134E mutant is a more active enzyme (Figure 4B,C) than S134K, suggesting that Glu134 has polar interactions with Arg126 and possibly Arg253, whereas Lys134 does not.

While Ser134 stabilizes PAP by forming a hydrogen bond with its 3'-phosphate (Figures 2A and 3A), its replacement with either glycine or alanine abolishes such hydrogen 


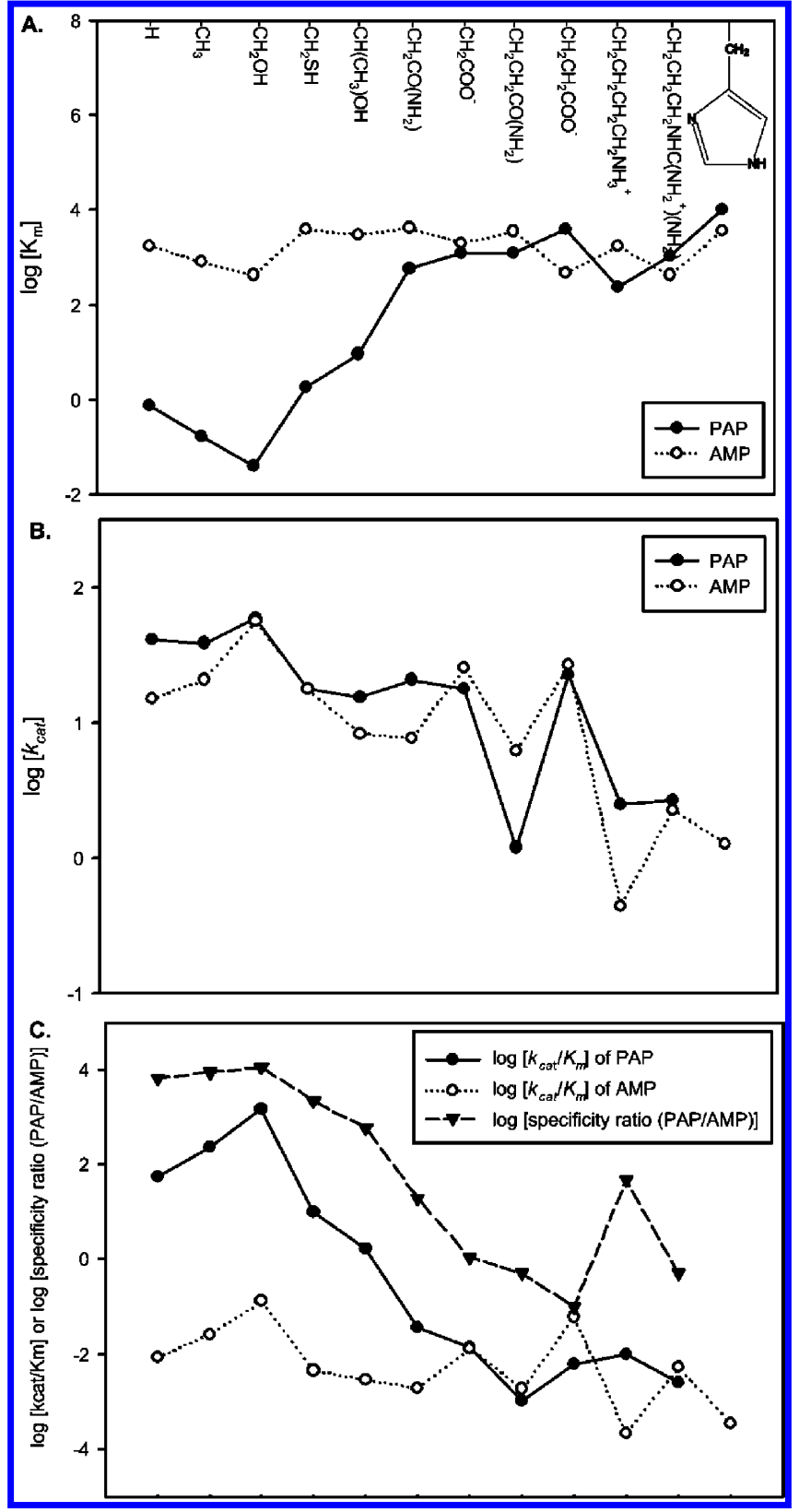

FIGURE 4: Impact of the mutation of Ser134 on the activity of PST. Data were derived from Table 4.

bonding. As a result, both S134G and S134A have higher $K_{\mathrm{m}}$ and lower $k_{\text {cat }}$ values than the wild type (Figure 4A,B) for both AMP and PAP.

Those mutants containing a bulky polar side chain at position 134 tend to alter the specificity ratio in favor of AMP (Figure 4), as $K_{\mathrm{m}}$ (Figure 4A) but not $k_{\text {cat }}$ (Figure 4B) is the dominant factor for this conversion. As shown in Figure $3 \mathrm{C}$, the presence of a bulky residue at position 134 does not appear to destroy the structure of the active site accommodating AMP but interferes with the binding of PAP because it occupies the presumed site of the 3'-phosphate of PAP. Ser134 has also been suggested to be critical for PAPS binding and catalysis $(19,23)$. We thus conclude that Ser134 plays an important role in nucleotide binding but not in catalysis. Also, Ser134 plays a key role in the determination of nucleotide specificity.

In contrast, the presence of a bulky hydrophobic residue at position 134 significantly disturbs the PST active site
(Figure 3A) and drastically reduces the enzymatic activity. Eight of 20 amino acids introduced at position 134 of PST show extremely low activity (Tables 2 and 4). All eight mutants (S134Y, S134W, S134F, S134M, S134P, S134V, S134I, and S134L) contain a bulky hydrophobic side chain.

Mutation of Arg126 to Ala proved to have a much more drastic effect on $K_{\mathrm{m}(\mathrm{PAP})}(5$ order of magnitude increase, Table 4) than that of $\operatorname{Arg} 253$, as a consequence of the latter guanidinium group interacting with the side chain of Tyr189 (Figure 3A). Since the side chain of Tyr 189 hydrogen bonds to the adenine of either AMP or PAP, destabilization of Tyr189 directly affects nucleotide binding. Mutation at position 254 is found to be less influential than that of positions 253 and 255, even though all three residues provide backbone interactions with the 3'-phosphate of PAP.

Mutation of Lys44, which directly interacts with the 5'phosphate of PAP, to Ala and Arg leads to the formation of exclusively the $\alpha$ form and predominantly the $\beta$ form of PST, respectively (Table 2). Mutation of Lys44 to Arg possibly results in the formation of two hydrogen bonds between Arg44 and the 5'-phosphate of PAP. Moreover, since Lys44 is located among Tyr135, Phe138, and Phe251, its mutation to Arg may also introduce additional interaction between its side chain and that of Phe138 (Figure 2B). By comparison, the K44A mutant not only abolishes the existing hydrogen bonds between Lys44 and the 5 '-phosphate but also generates a gap in the hydrophobic pocket comprising Pro43, Tyr135, Phe138, and Phe251.

Last, the PST model reveals residues directly interacting with a substrate. This study elucidates the role of each residue in the active site. Ser134 and $\operatorname{Arg} 126$, the side chains of which directly interact with the 3'-phosphate of PAP, influence the nucleotide selectivity of PST.

\section{ACKNOWLEDGMENT}

We thank Drs. William B. Jakoby and A. David Marshall for their generous gift of the pET3c11 plasmid and Dr. Farhad Forouhar for helpful suggestions.

\section{REFERENCES}

1. Jakoby, W. B., and Ziegler, D. M. (1990) J. Biol. Chem. 265, 20715-20718.

2. Falany, C. N. (1997) FASEB J. 11, 206-216.

3. Falany, J. L., and Falany, C. N. (1997) Oncol. Res. 9, 589-596.

4. Kotov, A., Falany, J. L., and Falany, C. N. (1999) J. Steroid Biochem. Mol. Biol. 68, 137-144.

5. Weinshilboum, R., and Otterness, D. (1994) in Toxicity (Kaufmann, F. C., Ed.) pp 45-78, Springer-Verlag, Berlin.

6. Varin, L., Marsolais, F., Richard, M., and Rouleau, M. (1997) FASEB J. 11, 517-525.

7. Kakuta, Y., Carter, C. W., Negish, M., and Pedersen, L. C. (1997) Nat. Struct. Biol. 4, 904-908.

8. Bidwell, L. M., McManus, M. E., Gaedigk, A., Kakuta, Y., Negishi, M., Pedersen, L., and Martin, J. L. (1999) J. Mol. Biol. 293, 521-530.

9. Dajani, R., Cleasby, A., Neu, M., Wonacott, A. J., Jhoti, H., Hood, A. M., Modi, S., Hersey, A., Taskinen, J., Cooke, R. M., Manchee, G. R., and Coughtrie, M. W. (1999) J. Biol. Chem. 274, 3786237868.

10. Kakuta, Y., Sueyoshi, T., Negishi, M., and Pedersen, L. C. (1999) J. Biol. Chem. 274, 10673-10676.

11. Perdersen, L. C., Petrotchenko, E. V., and Negishi, M. (2000) FEBS Lett. 475, 61-64

12. Driscoll, W. J., Komatsu, K., and Strott, C. (1995) Proc. Natl. Acad. Sci. U.S.A. 92, 12328-12332.

13. Marsolais, F., and Varin, L. (1995) J. Biol. Chem. 270, 3045830463. 
14. Marsolais, F., Laviolette, M., Kakuta, Y., Negishi, M., Pedersen, L. C., Auger, M., and Varin, L. (1999) Biochemistry 38, 40664071.

15. Sakakibara, Y., Takami, Y., Nakayama, T., Suiko, M., and Liu, M.-C. (1998) J. Biol. Chem. 273, 6242-6247.

16. Liu, M. C., Suiko, M., and Sakakibara, Y. (2000) J. Biol. Chem. 275, 13460-13464.

17. Kakuta, Y., Petrotchenko, E. V., Pedersen, L. C., and Negishi, M. (1998) J. Biol. Chem. 273, 27325-27330.

18. Petrotcheoko, E., Doerflein, M. E., Kakuta, Y., Pedersen, L. C., and Negishi, M. (1999) J. Biol. Chem. 274, 30019-30022.

19. Ong, E., Yeh, J.-C., Hindsgaul, O., Pedersen, L. C., Negishi, M., and Fukuda, M. (1999) J. Biol. Chem. 274, 25608-25612.

20. Lin, E. S., and Yang, Y. S. (2000) Biochem. Biophvs. Res. Commun. 271, 818-822.

21. Yang, Y. S., Marshall, A. D., McPhie, P., Guo, W. X., Xie, X. Chen, X., and Jakoby, W. B. (1996) Protein Expression Purif. 8, 423-429.

22. Lin, E. S., and Yang, Y. S. (1998) Anal. Biochem. 262, 111117.

23. Negishi, M., Pedersen, L. G., Petrotchenko, S. S., Gorokhov, A., Kakuta, Y., and Pedersen, L. C. (2001) Arch. Biochem. Biophvs. 390, 149-157.

BI0261239 